\title{
Qualidade de vida dos fisioterapeutas frente ao cenário imposto pela pandemia de COVID-19
}

\author{
Physiotherapists' quality of life in the face of the scenario imposed by the COVID-19 pandemic \\ Calidad de vida de los fisioterapeutas ante el escenario impuesto por la pandemia de COVID-19
}

Recebido: 26/11/2021 | Revisado: 03/12/2021 | Aceito: 05/12/2021 | Publicado: 16/12/2021

Fernanda Jesus da Silva

ORCID: https://orcid.org/0000-0002-3044-3596 Universidade Sociedade Educacional de Santa Catarina, Brasil E-mail: fisiofernanda@yahoo.com.br

Gideane Borges Gai

ORCID: https://orcid.org/0000-0003-1184-2855 Universidade Sociedade Educacional de Santa Catarina, Brasil

E-mail: gideanegai@gmail.com

Priscila Cembranel

ORCID: https://orcid.org/0000-0002-9560-686X Universidade Sociedade Educacional de Santa Catarina, Brasil E-mail: priscila_cembranel@yahoo.com.br

Luciane Taschetto

ORCID: https://orcid.org/0000-0002-0889-629X Universidade Sociedade Educacional de Santa Catarina, Brasil E-mail: luciane_taschetto@yahoo.com.br

Giselle Domingos

ORCID: https://orcid.org/0000-0001-6712-3476 Universidade Sociedade Educacional de Santa Catarina, Brasil E-mail: gigidomingos@ hotmail.com

\begin{abstract}
Resumo
A pandemia de SARS-CoV-2 afetou a qualidade de vida (QV) dos profissionais de saúde. Este estudo verifica a qualidade de vida dos fisioterapeutas que trabalham nos hospitais de Santa Catarina desde o início da pandemia da COVID-19. Trata-se de um estudo de abordagem quantitativa e descritiva, procedimento de pesquisa de levantamento e técnica de questionário fechado. É utilizado o QWLQ-bref, uma versão abreviada de um instrumento que visa aferir a QVT em quatro domínios: físico, psicológico, pessoal e profissional de fisioterapeutas que atuam em hospitais de um município catarinense. Os resultados da pesquisa evidenciam que no domínio físico a qualidade de vida apresentou uma baixa considerável nos quesitos: sono, necessidades fisiológicas e ambiente de trabalho. Já no domínio psicológico os respondentes sinalizaram estar mais inseguros, porém mais orgulhosos da profissão. No domínio pessoal, há um aumento nos quesitos relacionados às: relações ao trabalho, família e respeito pelos colegas. Em relação ao domínio profissional, a qualidade de vida é classificada como baixa e muito baixa. Assim, conclui-se que durante esse período de pandemia houve uma queda na qualidade de vida dos profissionais de fisioterapia que trabalham em instituições hospitalares.
\end{abstract}

Palavras-chave: Qualidade de vida; Fisioterapia; COVID-19; Qualidade de Vida no Trabalho (QVT).

\begin{abstract}
The SARS-CoV-2 pandemic affected the quality of life $(\mathrm{QL})$ of healthcare professionals. This study verifies the quality of life of physical therapists who have been working in hospitals in Santa Catarina since the beginning of the COVID-19 pandemic. This is a study with a quantitative and descriptive approach, survey research procedure and closed questionnaire technique. The QWLQ-bref is used, an abbreviated version of an instrument that aims to measure QWL in four domains: physical, psychological, personal and professional of physiotherapists working in hospitals in a city in Santa Catarina. The research results show that in the physical domain, quality of life had a considerable drop in the following items: sleep, physiological needs and work environment. In the psychological domain, respondents indicated that they were more insecure, but more proud of their profession. In the personal domain, there is an increase in items related to: work relationships, family and respect for colleagues. Regarding the professional domain, quality of life is classified as low and very low. Thus, it is concluded that during this period of pandemic there was a drop in the quality of life of physiotherapy professionals working in hospital institutions.
\end{abstract}

Keywords: Quality of life; Physiotherapy; COVID-19; Quality of Life at Work (QVT). 


\section{Resumen}

La pandemia de SARS-CoV-2 afectó la calidad de vida (QL) de los profesionales de la salud. Este estudio verifica la calidad de vida de los fisioterapeutas que trabajan en los hospitales de Santa Catarina desde el inicio de la pandemia COVID-19. Se trata de un estudio con enfoque cuantitativo y descriptivo, procedimiento de investigación por encuesta y técnica de cuestionario cerrado. Se utiliza el QWLQ-bref, una versión abreviada de un instrumento que tiene como objetivo medir la QWL en cuatro dominios: físico, psicológico, personal y profesional de fisioterapeutas que trabajan en hospitales de una ciudad de Santa Catarina. Los resultados de la investigación muestran que en el dominio físico, la calidad de vida presentó una caída considerable en los ítems: sueño, necesidades fisiológicas y ambiente laboral. En el ámbito psicológico, los encuestados indicaron que se sentían más inseguros, pero más orgullosos de su profesión. En el ámbito personal, se incrementan los ítems relacionados con: relaciones laborales, familia y respeto a los compañeros. En cuanto al dominio profesional, la calidad de vida se clasifica en baja y muy baja. Así, se concluye que durante este período de pandemia hubo una disminución en la calidad de vida de los profesionales de fisioterapia que laboran en instituciones hospitalarias.

Palabras clave: Calidad de vida; Fisioterapia; COVID-19; Calidad de vida en el trabajo (QVT).

\section{Introdução}

A Qualidade de Vida no Trabalho (QVT), segundo a Organização Mundial da Saúde (OMS), pode ser definida como "percepção do indivíduo de sua posição na vida no contexto da cultura e sistemas de valores nos quais ele vive e em relação aos seus objetivos, expectativas, padrões e preocupações". (Santana et al., 2014, p. 35). Trata-se de uma concepção ampla que abrange tanto o lado psicológico como o lado físico (Andrade et al., 2018).

"Qualidade de Vida" é o termo utilizado para avaliar os processos multifatoriais que definem o limiar entre saúde e doença. Tal mensuração é feita por meio de instrumentos simples e complexos. Os simples dizem respeito ao registro sistematizado de observação e os complexos aos questionários testados estatisticamente. Muitos instrumentos complexos podem dar lugar às avaliações simples sobre a satisfação pessoal e profissional. Porém o conceito de QVT é melhor verificado quando os instrumentos são complexos, pois permitem a observação de mais aspectos da observação (Pereira et al., 2012).

Devido ao início da pandemia de SARS-CoV-2 a análise de QV se utiliza do questionário proposto por Reis Jr et al. (2011), chamado de QWLQ-78. Este, é baseado no WHOQOL-100 desenvolvido pela Organização Mundial da Saúde (OMS) e reorganizado em menos questões para facilitar seu preenchimento. Criaram-se assim quatro domínios: físico/saúde, psicológico, pessoal e profissional (Reis Jr et al., 2011).

O profissional de fisioterapia, como parte da equipe de saúde, tem desempenhado esforços para garantir a segurança de pacientes e familiares. No entanto, com todas as medidas de segurança em seu entorno não é possível garantir que sua QVT seja preservada ou melhorada desde o início da pandemia (Oliveira et al., 2020). Pois, acredita-se que o desgaste dos profissionais influencie nos atendimentos quando há alterações nos quesitos de QVT. No entanto, esse impacto pode ser minimizado por meio de diagnóstico dos profissionais de fisioterapia. Um bom diagnóstico dos aspectos sociais, econômicos e individuais pode mitigar os riscos e proporcionar a adoção de ações preventivas e de tratamento, como por exemplo a busca por suporte emocional e melhor compressão da situação (Esperidião et al., 2020).

O conceito de Qualidade de Vida (QV) é estudado em diferentes áreas de conhecimento e varia conforme contexto e momento histórico. Inicialmente, a QV relacionava-se à condição de vida e ao poder aquisitivo de um indivíduo, abordagem que se tornou insuficiente com o passar do tempo. Pois, o aumento da expectativa de vida trouxe mais aspectos a serem considerados na avaliação, como a condição de saúde dos indivíduos, por exemplo (Cruz et al., 2018).

Segundo a OMS (1995), QV é a forma como o indivíduo visualiza a sua condição de vida e inclui aspectos como: contexto, cultura, valores, objetivos, desejos e medos. Sabe-se, no entanto, que a análise dessa percepção é subjetiva e afetada pela forma como a pessoa se relaciona com as diferentes dimensões da própria vida (Paz et al., 2020). 
O conceito de QV é absorvido pelo conceito de QVT. Assim, entende-se como Qualidade de Vida no Trabalho (QVT) o completo desenvolvimento das potencialidades físicas e intelectuais, juntamente ao bem-estar físico, mental, material e social, respeitando-se os princípios de segurança, higiene e ergonomia (Spiller et al., 2008).

Pelo viés da empresa, a QVT pode ser compreendida como: "O conjunto de ações desenvolvidas pelas empresas na implantação de melhoria gerenciais, estruturais e tecnológicas, na busca da satisfação e do bem-estar físico, psicológico, social e profissional dos colaboradores" (Reis Jr., 2008, p. 57-58). O conceito pode ser compreendido ainda, como a capacidade de resposta dos trabalhadores às demandas laborais, tais como: estresse, engajamento e bem-estar no trabalho (Craigie, 2016).

A QVT é fundamental para o atendimento das necessidades dos trabalhadores e tem o objetivo de humanizar o trabalho e trazer satisfação, bem-estar e participação em decisões nas empresas (Sampaio, 2012).

O questionário QWLQ-78, QWLQ-bref é considerado um questionário de QVT completo e, por isso utilizado pela maioria dos pesquisadores (Reis Jr., 2008). Devido ao seu prestígio, é utilizado para apresentar resultados e representações sobre a QVT dos indivíduos, sendo possível ser usado em diversos tipos de população e empresas (Reis Jr et al., 2011). O QWLQ-bref é a versão abreviada do QWLQ-78.

Figura 1: Domínios do QWLQ.

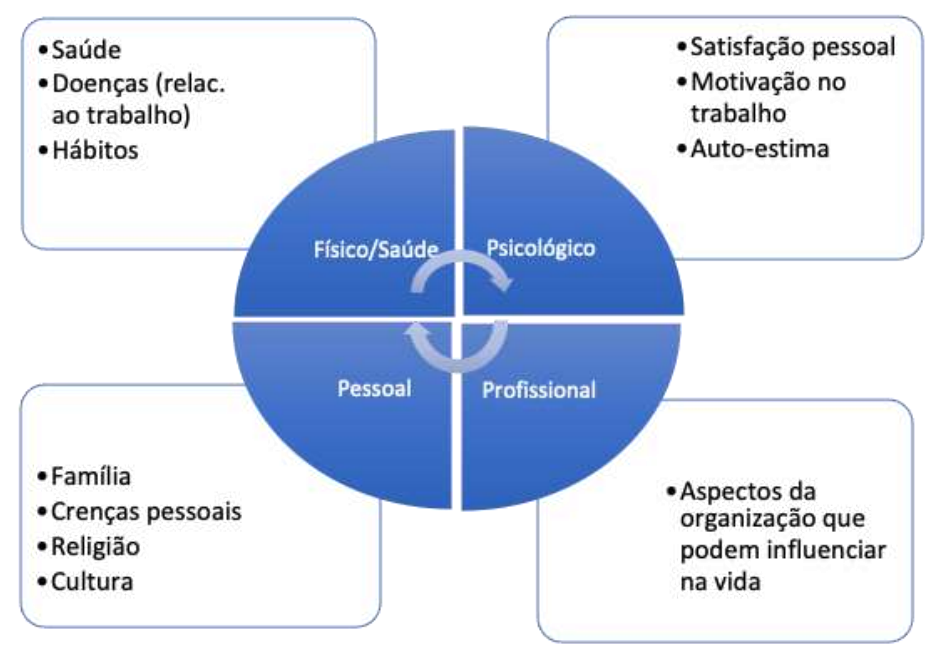

Fonte: Adaptado de Reis Jr. (2008).

O QWQL-78 possui quatro domínios: aspectos físicos, relacionamentos sociais, ambientais e psicológicos (Figura 1). O número de questões por domínio é diferente. São 17 questões sobre aspectos físicos/saúde, 10 sobre aspectos psicológicos, 16 sobre os aspectos pessoais e 35 sobre os profissionais alocadas de forma aleatória. As respostas são fornecidas por meio de escala Likert de cinco pontos que pode surgir da seguinte forma:

1 Nada, 2 muito pouco, 3 mais ou menos, 4 bastante, 5vextremamente;

1 Muito baixa, 2 baixa, 3 média, 4 alta, 5 muito alta; istep:

1 Nunca, 2 raramente, 3 às vezes, $\mathrm{f} 4$ frequentemente, 5 sempre;

1 Muito ruim, 2 ruim, 3 médio, 4 bom, 5 muito bom (REIS JÚNIOR, 2008, p. XX).

Em relação aos aspectos físicos, são abordados os temas: dor física, atividades diárias, sono, fadiga, capacidade e dependência do trabalho. Deve-se levar em conta que muitas perspectivas podem influenciar nos aspectos físicos, por exemplo: uma pessoa com autoestima baixa pode ter seus aspectos físicos prejudicados e essa informação deve ser considerada na 
avaliação final (Mendonça et al., 2015). As perguntas que abordam esse assunto devem ser voltadas às doenças, ao trabalho e hábitos de vida (Reis Jr et al., 2011).

No domínio psicológico consideram-se os temas: afetos, memória, concentração, imagem corporal, autoestima e aparência (Damásio et al., 2013). Para esse campo de avaliação, buscam-se soluções que possam compreender as reações de um indivíduo e suas vivências. Os aspectos psicológicos indicam como os entrevistados percebem suas vidas, felicidade e satisfação (Pereira et al., 2012).

Em terceiro lugar, têm-se o domínio social que aborda os aspectos familiares, crenças religiosas e aspectos culturais (Reis Jr et al., 2011). Esse tema pode ser entendido como a percepção do indivíduo sobre seus relacionamentos tanto sociais quantos pessoais (Gordia et al., 2015).

Por último existem os aspectos profissionais que englobam questões sobre a segurança física, segurança dos recursos financeiros, moradia, transporte, entre outros (Damásio et al., 2013).

Os resultados são avaliados conforme a pontuação. Se o índice estiver entre $0-22,5 \%$ a QVT é considerada muito insatisfatória, se estiver entre 22,5 - 45 é considerada insatisfatória, entre 45 - 55 neutra, entre 55 - 75 satisfatória e 77,5 - 100 é considerada muito satisfatória (Reis Jr., 2008).

O QWLQ-bref é a versão abreviada do QWLQ-78. Fazem parte dessa versão apenas um quarto das questões de cada domínio. Ao total são 4 questões sobre domínio pessoal, três sobre domínio psicológico, quatro sobre domínio físico/saúde e nove sobre domínio profissional. O objetivo de encurtar o questionário foi a economia de tempo e a facilitação para tabulação dos dados (Chemereta et al., 2011).

Durante pandemias é comum que profissionais de saúde trabalhem horas seguidas, sem pausas, sem descanso e sob grande pressão. Essa situação leva os profissionais ao esgotamento, pois as longas jornadas diminuem o nível de atenção e a capacidade de resposta interferindo na qualidade do cuidado a saúde e na imunidade do profissional (Helioterio et al., 2020).

É comum que o aumento da jornada de trabalho interfira nas relações sociais dos profissionais. Além da saúde física, é aumentada a possibilidade de os profissionais desenvolverem problemas psicológicos causados pela situação de medo e incerteza (Spiller, Dyniewicz \& Slomp, 2008).

Diante de uma situação de isolamento também é comum que as pessoas se sintam menos satisfeitas e motivadas (Schramm et al., 2020). Os impactos psicológicos relacionam-se com diversos setores da vida. De acordo com Ornell et al. (2020, p. 2) há mudanças na “organização familiar, fechamento de escolas, empresas e locais públicos, mudanças nas rotinas de trabalho, isolamento, levando a sentimentos de desamparo e abandono". É comum que as pessoas se sintam temerosas pela economia e por estarem vivendo em menor convívio social.

Hábitos foram modificados, lavar as mãos com água e sabão, uso de álcool em gel, protocolos de isolamento, uso de Equipamentos de Proteção Individual (EPIs) específicos para os profissionais da saúde (avental, capote, máscara No 95, máscara cirúrgica, óculos, gorro, proteção descartável para sapatos e luvas) e máscaras cirúrgicas descartáveis ou laváveis para a população em geral. Foram apenas algumas das mudanças de hábitos adotadas desde o início da pandemia (Soeiro et al., 2020). Até mesmo os hábitos de consumo antes presenciais, também migraram para o mercado online.

A discussão sobre a valorização dos profissionais da saúde, dentre eles o fisioterapeuta, tornou-se ainda mais pertinente após o início da pandemia de SARS-CoV-2, pois muitas foram as alterações em termos de jornada de trabalho, sobrecarga, insegurança e até mesmo diminuição de pacientes (Helioterio et al., 2020; Karsten et al., 2020).

Em tempos de pandemia, o atendimento aos pacientes internados no ambiente hospitalar pode comprometer a saúde física e mental dos profissionais da saúde. A isso, somam-se as inseguranças causadas pelo enfrentamento de uma doença não conhecida em todos seus aspectos. Essa nova realidade pode interferir na qualidade de vida do profissional que teve que alterar drasticamente sua rotina (Santana et al., 2014; Helioterio et al., 2020). 
Diante de tal situação, a QVT pode e deve ser analisada. E, uma das formas é por meio da utilização de ferramentas como questionários, tais como: WHOQOL-bref uma versão abreviada do WHOQOL-100, QWLQ-bref a versão abreviada do QWLQ-78, o modelo de Walton, modelo de Westley, modelo de Hackman e Oldham, modelo de Werther e Davis, modelo Nadler e Lawler, o instrumento TQWL-42. Esses instrumentos foram desenvolvidos no intuito de medir a QV no trabalho (Ferro, 2012).

Com tantas mudanças nos protocolos e as dificuldades causadas pelo desconhecimento, este trabalho busca resolver o seguinte problema: “Como está a Qualidade de Vida dos fisioterapeutas desde o início da pandemia da COVID-19?”. Deste modo, será possível verificar a QVT dos fisioterapeutas que trabalham em dois hospitais de um município do norte catarinense desde que foi decretada a pandemia de COVID-19".

\section{Metodologia}

Esta pesquisa foi desenvolvida por meio de abordagem quantitativa e descritiva, procedimento de pesquisa de levantamento e técnica de questionário fechado via survey.

O presente estudo foi realizado com os 15 fisioterapeutas que trabalham em nos dois hospitais de um município do norte catarinense.

A coleta de dados foi realizada no mês de setembro e outubro de 2020, onde foi enviado um link hospedado no Microsoft Forms do questionário survey aos participantes. A pesquisa foi realizada mediante a aplicação da ferramenta conhecida como QWLQ-bref. A análise de dados foi desenvolvida por meio da técnica de análise estatística de frequência.

Para efeitos de análise, foram elencadas duas escalas Likert com cinco pontos. Uma a respeito da QVT antes do surgimento da pandemia e outra depois, focando especialmente nas últimas duas semanas antes de responder ao questionário.

Foram analisados pelos participantes quatro domínios: físico, psicológico, pessoal e profissional. O domínio físico abordou variáveis a respeito de saúde, doenças relacionadas ao trabalho e hábitos, o domínio psicológico contou com variáveis a respeito da satisfação pessoal, motivação no trabalho e autoestima. O domínio pessoal abarca os tópicos família, crenças pessoais, religião e cultura e, por fim o domínio profissional avalia aspectos organizacionais que podem influenciar na vida dos respondentes.

As informações com as respostas serão apresentadas em forma de tabela com os respectivos percentuais. Para avaliação e comparação será considerado o maior percentual em cada critério (antes ou depois da pandemia).

\section{Resultados e Discussão}

Participaram deste estudo quinze fisioterapeutas que trabalham em dois hospitais da região norte de Santa Catarina, Brasil. Destes, quatro são homens e onze mulheres. A idade média geral dos respondentes é de vinte e oito anos.

Em relação ao tempo de atuação, a média é de sessenta meses dedicados pelos fisioterapeutas em seu trabalho na área hospitalar.

O domínio físico possui quatro perguntas. A primeira busca avaliar o sono, a segunda aborda os problemas causados pelo sono no trabalho, a terceira questiona se as necessidades fisiológicas básica são satisfeitas adequadamente e a quarta examina o conforto no ambiente de trabalho. Para melhor visualização nas tabelas os percentuais foram arredondados. 
Quadro 1: Domínio físico.

\begin{tabular}{|c|c|c|c|c|c|c|}
\hline QUESTÃO & Critério & $\begin{array}{ll}1 & (\mathrm{em} \\
\%)\end{array}$ & $\begin{array}{ll}2 & (\mathrm{em} \\
\%) & \end{array}$ & $\begin{array}{l}3 \quad(\mathrm{em} \\
\%)\end{array}$ & $\begin{array}{l}4 \quad(\mathrm{em} \\
\%)\end{array}$ & $\begin{array}{ll}5 & (\mathrm{em} \\
\%) & \end{array}$ \\
\hline \multirow[t]{2}{*}{ Em que medida você avalia o seu sono? } & ANTES & & & 20 & 60 & 20 \\
\hline & DEPOIS & 7 & 13 & 53 & 27 & \\
\hline \multirow{2}{*}{$\begin{array}{l}\text { Em que medida algum problema com o sono prejudica } \\
\text { seu trabalho? }\end{array}$} & ANTES & 7 & 47 & 33 & 7 & 7 \\
\hline & DEPOIS & & 27 & 40 & 33 & 7 \\
\hline \multirow{2}{*}{$\begin{array}{c}\text { Suas necessidades fisiológicas básicas são satisfeitas } \\
\text { adequadamente? }\end{array}$} & ANTES & & & 33 & 60 & 7 \\
\hline & DEPOIS & & 27 & 40 & 27 & 7 \\
\hline \multirow{2}{*}{$\begin{array}{l}\text { Em que medida você se sente confortável no ambiente } \\
\text { de trabalho? }\end{array}$} & ANTES & & 7 & 7 & 73 & 13 \\
\hline & DEPOIS & 7 & 13 & 33 & 40 & 7 \\
\hline
\end{tabular}

Fonte: Dados da própria pesquisa (2020).

Ao avaliarem o sono, os respondentes demonstraram que antes da pandemia dormiam melhor, como pode ser observado no Quadro 1. Entretanto, após a pandemia, 71,4\% dos respondentes sinalizaram ter problemas relacionados ao sono.

No cotidiano, o sono está ligado a diversos fatores que podem interferir diretamente em aspectos como: o humor, memória, atenção, registros sensoriais, raciocínio, e nos aspectos cognitivos (Valle, Valle \& Reimão, 2009). Por isso, indivíduos que dormem menos que o necessário sofrem uma diminuição do seu vigor físico, envelhecimento precoce, vulnerabilidade a infecções, obesidade, hipertensão e diabetes (Ravagnani \& Crivelato, 2010). E, com os números de casos de COVID-19 aumentando, a demanda dos profissionais é cada vez maior. Isso fez com que a correria do dia a dia forçasse os profissionais a ignorassem as próprias necessidades e dediquem tempo e energia para tratar pacientes. (Teixeira et al., 2020).

Ao analisarem o bem-estar no ambiente de trabalho, antes da pandemia este aspecto era considerado por $73 \%$, muito confortável. Para o segundo momento, esse percentual caiu para 40\%, segundo os respondentes.

Os profissionais relatam o alto risco de infecção e a proteção inadequada se juntam ao excesso de trabalho, discriminação, frustração, assistência a pacientes com emoções negativas e a falta de contato com a família e exaustão. Esse acúmulo de estímulos, sensações e sentimentos causam problemas de saúde mentais e emocionais. Diante disso, é comum observar dificuldade de prestar atenção, entender instruções e tomar decisões rápidas. Esse conjunto de situações acabam afetando o bem-estar geral (Teixeira et al., 2020).

O domínio físico possui três perguntas. A primeira busca avaliar a motivação, a segunda a liberdade de expressão no trabalho e a terceira o orgulho da profissão, como pode ser observado no Quadro 2.

Quadro 2: Domínio psicológico.

\begin{tabular}{|c|c|c|c|c|c|c|}
\hline QUESTÃO & Critério & $\begin{array}{l}1 \quad(\mathrm{em} \\
\%)\end{array}$ & $\begin{array}{ll}2 & (\mathrm{em} \\
\%) & \end{array}$ & $3(\mathrm{em} \%)$ & $\begin{array}{l}4 \\
(\mathrm{em} \%)\end{array}$ & $\begin{array}{ll}5 & (\mathrm{em} \\
\%) & \end{array}$ \\
\hline \multirow{2}{*}{$\begin{array}{l}\text { Em que medida você̂ avalia sua motivação para } \\
\text { trabalhar? }\end{array}$} & ANTES & & 13 & 20 & 60 & 7 \\
\hline & DEPOIS & 7 & 13 & 27 & 33 & 20 \\
\hline \multirow{2}{*}{$\begin{array}{l}\text { Como você avalia sua liberdade de expressão no seu } \\
\text { trabalho? }\end{array}$} & ANTES & & 7 & 47 & 20 & 27 \\
\hline & DEPOIS & 7 & 13 & 27 & 33 & 20 \\
\hline \multirow{2}{*}{$\begin{array}{l}\text { Em que medida você avalia o orgulho pela sua } \\
\text { profissão? }\end{array}$} & ANTES & & & 7 & 47 & 47 \\
\hline & DEPOIS & & & & 7 & 93 \\
\hline
\end{tabular}

Fonte: Dados da própria pesquisa (2020). 
Os fisioterapeutas, em sua maioria, avaliaram a motivação para o trabalho como alta antes da pandemia. Percebe-se uma mudança nesses percentuais quando questionados a respeito de suas últimas duas semanas de trabalho. Apesar de o maior percentual de respondentes ainda afirmar que continua motivado, percebe-se que a segunda resposta mais citada foi "média". Isso pode acontecer devido a mudança constante de orientações e a sobrecarga de trabalho (Antunes et al., 2013).

Em relação a liberdade de expressão e ao orgulho no desempenho da profissão, é possível visualizar um aumento nesses percentuais após a pandemia.

Silva e Souza (2020) relatam que durante a pandemia de COVID-19 a fisioterapia teve grande importância na recuperação dos sintomas como a Síndrome Respiratória Aguda (SRA). Além dos aspectos respiratórios, alguns sintomas podem ser de ordem neurológica, gastrointestinal e musculoesquelético. Todas essas questões podem ser tratadas pelo fisioterapeuta, que vem sendo solicitado cada vez mais precocemente. Essa procura faz com que o profissional se sinta útil e visível sociedade também.

O domínio pessoal possui quatro perguntas. A primeira aborda a realização pessoal com o trabalho, a segunda avalia a qualidade da relação com superiores e subordinados, a terceira mensura a avaliação da família em relação ao trabalho do fisioterapeuta e a quarta busca conhecer o respeito entre colegas e superiores no trabalho, como pode ser observado no Quadro 3.

Quadro 3: Domínio pessoal.

\begin{tabular}{|c|c|c|c|c|c|c|}
\hline QUESTÃO & Critério & $1(\mathrm{em} \%)$ & $\begin{array}{ll}2 & (\mathrm{em} \\
\%) & \end{array}$ & $3(\mathrm{em} \%)$ & $\begin{array}{l}4 \\
(\mathrm{em} \%)\end{array}$ & $\begin{array}{ll}5 & (\mathrm{em} \\
\%) & \end{array}$ \\
\hline \multirow[t]{2}{*}{ Você se sente realizado com o trabalho que faz? } & ANTES & & & 20 & 53 & 27 \\
\hline & DEPOIS & & & 20 & 40 & 40 \\
\hline \multirow{2}{*}{$\begin{array}{l}\text { Como você̂ avalia a qualidade da sua relação com seus } \\
\text { superiores e/ou subordinados? }\end{array}$} & ANTES & & & 13 & 53 & 33 \\
\hline & DEPOIS & 7 & 7 & 20 & 33 & 33 \\
\hline \multirow[t]{2}{*}{ Em que medida sua família avalia seu trabalho? } & ANTES & & & 7 & 60 & 33 \\
\hline & DEPOIS & & & & 20 & 80 \\
\hline \multirow{2}{*}{$\begin{array}{c}\text { Em que medida vocề é respeitado pelos seus colegas e } \\
\text { superiores? }\end{array}$} & ANTES & & & 33 & 53 & 13 \\
\hline & DEPOIS & & & 27 & 53 & 20 \\
\hline
\end{tabular}

Fonte: Dados da própria pesquisa (2020).

Os profissionais em sua maioria foram positivos em relação a sua satisfação com o trabalho tanto antes como depois da pandemia. Mais da metade afirma que está "muito" e "completamente" satisfeito com o trabalho que fazem. Quando analisados os percentuais dos dois itens antes da pandemia e depois, pode-se afirmar que houve um aumento médio no índice de satisfação dos respondentes.

A profissão do fisioterapeuta passou a ter destaque desde o início da infecção da COVID-19. Isso aconteceu em um momento em que a doença e seus tratamentos eram pouco conhecidos e trouxe a valorização das atividades do profissional nos hospitais (Sumiya, 2020). Este passou a ser mais valorizado, reconhecido e solicitado junto as equipes profissionais.

Em relação a qualidade das relações com superiores e subordinados antes da pandemia, metade dos fisioterapeutas, classificaram está como "alta". No entanto, desde que as coisas se intensificaram nos hospitais, é possível afirmar que a qualidade das relações foi afetada, tendo em vista os $13 \%$ que classificaram estas como "muito baixas" e "baixas".

Nesse primeiro de pandemia houveram situações de estresse, exaustão, transtornos de ansiedade e o esgotamento dos trabalhadores. E, as equipes de profissionais batalharam mesmo diante da gravidade dos casos, da falta de equipamentos, de EPI's, leitos e até mesmo equipamentos de suporte a vida (Teixeira et al., 2020). 
Sobre os familiares, mais da metade dos profissionais afirmaram que estes viam o seu trabalho como "bom". Reconhecimento que aumentou desde o início da pandemia.

Antes, a fisioterapia era mais voltada aos distúrbios ortopédicos traumatológicos e neurológicos. Após a repercussão e as mudanças sociais e epidemiológicas, a população passou a observar a intervenção dessa profissão na atenção primária e promoção da saúde, sem abandonar a parte da reabilitação. Outro ponto a ser salientado foi o atendimento domiciliar observando os devidos cuidados impostos pela pandemia (Assobrafir, 2020).

Outro aspecto avaliado foi o respeito recebido por colegas e superiores. Percebeu-se pelas respostas que as pessoas são "muito" respeitadas e isso não sofreu grande alteração. Entretanto, deve ser pontuada a melhoria no percentual de pessoas que passaram a perceber que são "completamente" respeitadas. Isso pode ocorrer devido ao bom prognóstico dos pacientes que necessitam de cuidados respiratórios avançados, pois as condutas exigem avaliações e reavaliações frequentes realizadas pelos fisioterapeutas (Socorro et al., 2020).

Cabe salientar também que esse reconhecimento e respeito observados podem ter relação ao reconhecimento social dos fisioterapeutas nas equipes de saúde. Estes passaram a ter envolvimento ativo na tomada de decisão, coesão, coordenação de cuidado e resolutividade de problemas. Pois, sem essa abordagem há uma considerável diminuição da efetividade dos resultados (Belarmino et al., 2020).

O domínio pessoal possui nove perguntas. A primeira avalia a liberdade para criar coisas novas, a segunda a igualdade de tratamento entre funcionários, a terceira mensura o orgulho da organização que o fisioterapeuta trabalha. Já a quarta fala da satisfação com a participação nas decisões da empresa, a quinta busca compreender o nível de responsabilidade no trabalho, a sexta avalia os treinamentos recebidos. Por conseguinte, a sétima fala sobre a satisfação em relação a variedade de tarefas realizadas, a oitava do espírito de camaradagem no trabalho e por fim, a nona sobre a satisfação com qualidade de vida no trabalho. Como pode ser observado no Quadro 4.

Quadro 4: Domínio profissional.

\begin{tabular}{|c|c|c|c|c|c|c|}
\hline QUESTÃO & Critério & $1(\mathrm{em} \%)$ & $\begin{array}{ll}2 & (\mathrm{em} \\
\%) & \end{array}$ & $\begin{array}{ll}3 & (\mathrm{em} \\
\%) & \\
\end{array}$ & $4(\mathrm{em} \%)$ & $\begin{array}{ll}5 & (\mathrm{em} \\
\%)\end{array}$ \\
\hline \multirow{2}{*}{$\begin{array}{l}\text { Como vocề avalia a sua liberdade para criar coisas } \\
\text { novas no trabalho? }\end{array}$} & ANTES & & 7 & 27 & 47 & 20 \\
\hline & DEPOIS & 13 & & 33 & 33 & 20 \\
\hline \multirow{2}{*}{$\begin{array}{l}\text { Como você avalia a igualdade de tratamento entre os } \\
\text { funcionários? }\end{array}$} & ANTES & 7 & 13 & 47 & 27 & 7 \\
\hline & DEPOIS & 13 & 7 & 47 & 20 & 13 \\
\hline \multirow{2}{*}{$\begin{array}{l}\text { Em que medida você̂ possui orgulho da organização na } \\
\text { qual trabalha? }\end{array}$} & ANTES & 7 & 7 & 20 & 53 & 13 \\
\hline & DEPOIS & 13 & & 13 & 53 & 20 \\
\hline \multirow{2}{*}{$\begin{array}{l}\text { Em que medida você̂ está satisfeito com o seu nível de } \\
\text { participação nas decisões da empresa? }\end{array}$} & ANTES & 13 & 20 & 27 & 33 & 7 \\
\hline & DEPOIS & 13 & & 27 & 53 & 7 \\
\hline \multirow{2}{*}{$\begin{array}{l}\text { Você está satisfeito com o seu nível de } \\
\text { responsabilidade no trabalho? }\end{array}$} & ANTES & & & 40 & 47 & 13 \\
\hline & DEPOIS & & 7 & 20 & 60 & 13 \\
\hline \multirow{2}{*}{$\begin{array}{l}\text { Vocề se sente satisfeito com os treinamentos dados } \\
\text { pela organização? }\end{array}$} & ANTES & & 33 & 33 & 20 & 13 \\
\hline & DEPOIS & 7 & 13 & 47 & 13 & 20 \\
\hline \multirow{2}{*}{$\begin{array}{l}\text { Vocề se sente satisfeito com a variedade das tarefas } \\
\text { que realiza? }\end{array}$} & ANTES & & & 33 & 60 & 7 \\
\hline & DEPOIS & & 20 & 20 & 47 & 13 \\
\hline \multirow{2}{*}{$\begin{array}{l}\text { Como vocề avalia o espírito de camaradagem no seu } \\
\text { trabalho? }\end{array}$} & ANTES & & 13 & 20 & 53 & 13 \\
\hline & DEPOIS & & 13 & 20 & 47 & 20 \\
\hline \multirow{2}{*}{$\begin{array}{l}\text { O quanto vocề está satisfeito com a sua qualidade de } \\
\text { vida no trabalho? }\end{array}$} & ANTES & & 13 & 40 & 40 & 7 \\
\hline & DEPOIS & 7 & 27 & 27 & 33 & 7 \\
\hline
\end{tabular}

Fonte: Dados da própria pesquisa (2020).

Os profissionais considerar que a sua liberdade para criar coisas novas no trabalho caiu desde o início da pandemia. Esta trouxe alguns protocolos rígidos e isso impactou na liberdade dos profissionais. Os profissionais da saúde que foram escalados exclusivamente para tratar de pacientes com sintomas da doença foram isolados de outros setores do hospital durante 
o horário do trabalho. Essa situação se intensificou com o aumento dos casos e mais EPI's foram incluídos nos protocolos (IBSP, 2020).

Em relação a igualdade de tratamento entre funcionários, pode-se afirmar que estes perceberam uma piora desde que a pandemia começou, já que esse percentual caiu: 7\% afirmaram como "baixa" e 13\% classificaram tal questão como "muito baixa”. Esse é um ponto de alerta, pois a igualdade de tratamento impacta diretamente na motivação das pessoas. Sabe-se também, que esse é um aspecto que afeta a produtividade e o atingimento das metas organizacionais (Carvalho et al., 2012).

Sobre o orgulho de trabalhar na organização atual, os fisioterapeutas demonstraram um pequeno aumento se for considerada a classificação "completamente". O que não altera o resultado significativamente, já que a amostra de respondentes era pequena.

O orgulho dos profissionais da saúde costuma ser percebido quando há o sentimento de contribuição com a sociedade e o reconhecimento dos serviços prestados. Ainda que seja um trabalho intenso e cansativo, ter a confiança dos pacientes traz o sentimento de valorização profissional dos membros das equipes multidisciplinares (Maissiat et al., 2015).

Os profissionais mensuraram sua satisfação com a participação nas decisões do hospital. Diante dos resultados, é possível observar se comparados os dois momentos, antes e depois da pandemia, houve um crescimento de $20 \%$ em termos de participação. Critério importante para trazer segurança às condutas profissionais adotadas, tendo em vista que estas afetam a vida, saúde, integridade físico-psíquica e relações sociais dos indivíduos (Araújo et al., 2008).

Em relação à satisfação com as responsabilidades no trabalho, houve uma pequena melhora em relação ao antes e ao depois da pandemia. No entanto, existe um percentual de 7\% que expressou uma baixa satisfação com esse aspecto apenas na pós pandemia. Fato que não recebeu pontuação no cenário pré-pandemia. Sobre esse tema percebeu-se que, muitos profissionais modificaram a jornada de trabalho e a forma como desempenham suas atividades para atender aos paciente. Isso, somado ao cenário de incerteza, aumentou também a preocupação com a própria segurança e a dos familiares (Ribeiro et al., 2020).

Os fisioterapeutas também responderam sobre treinamentos fornecidos pelos hospitais. Sobre isso podem ser feitas duas considerações. A primeira é a melhora da percepção dos respondentes acerca dos treinamentos. Percebeu-se que, na póspandemia houve um aumento de satisfação em relação aos treinamentos recebidos. A segunda é a satisfação média com os treinamentos, que também sofreu um aumento quando comparado o antes e o depois da pandemia.

Almeida (2020) afirma que, qualquer treinamento precisa chamar a atenção para o fato de que a pandemia evolui mais rápido do que as medidas de prevenção de novos riscos. Nesse contexto, os participantes devem estar cientes do ambiente incerto de trabalho para enfrentarem mudanças ocorridas nos protocolos e estarem preparados a fazerem alterações em suas condutas diante de nova evidência que possa ser relevante (Lotta et al., 2020).

Os respondentes também classificaram a variedade de tarefas realizadas. De modo geral, é possível inferir que as pessoas estavam mais satisfeitas antes da pandemia, pois a satisfação diminuiu.

Tal informação pode ser explicada devido aos fisioterapeutas trabalharem em hospitais e clínicas. Assim, se a atuação não se limita a área hospitalar, diversas são as abordagens possíveis, inclusive no pós-internação dos pacientes de COVID-19 (Guimarães, 2020).

Em relação ao espírito de camaradagem, os entrevistados declararam que houve melhora se comparados os momentos antes e depois da pandemia.

$\mathrm{Na}$ área da saúde, o conceito de equipe no ambiente hospitalar não se restringe apenas a um conjunto de indivíduos. Ele trata da multiplicidade de abordagens que conectam os processos de produção de saúde com vistas a melhorar a qualidade de atendimento (Peruzzo et al., 2018). 
Por fim, os fisioterapeutas mediram a satisfação geral em relação a QVT. Antes, consideravam esse aspecto de "baixa" satisfação. Após a pandemia, afirmaram que sua qualidade de vida poderia ser classificada como "muito baixa" e "baixa".

Um estudo realizado sobre saúde mental da pandemia da COVID-19 apontou alguns sintomas que exemplificam o prejuízo para os trabalhadores da área da saúde. Dentre eles podem ser listados: a sensação de alto risco de contaminação, a perda de qualidade de vida profissional e o aumento de alterações de humor (Faro et al., 2020).

Do mesmo modo, grande parte dos profissionais sofreram impactos na saúde mental após epidemias e pandemias. Além disso, a maior parte destes também sofreram algum tipo de dano físico decorrente do excesso de trabalho (Silva et al. 2020).

Assim, é possível perceber que a qualidade de vida dos profissionais vem sendo abalada. Estes, trabalham e estudam sobre a doença para ter alternativas plausíveis nos tratamentos que administram. Isso faz com que exista uma sobrecarga e um maior nível de responsabilidade diante dos protocolos que ainda não estão consolidados. Por esse motivo, é importante que os profissionais tenham apoio psicológico para cuidar da sua saúde mental e manter o mínimo de qualidade de vida em meio ao caos que estão vivendo (Faro et al., 2020).

\section{Considerações Finais}

O presente estudo identificou as mudanças em termos de qualidade de vida por meio da utilização do questionário QWLQ-78-bref.

Assim, em relação ao objetivo geral: "Verificar a qualidade de vida dos fisioterapeutas que trabalham nos hospitais desde o início da pandemia da COVID-19”, a coleta de dados realizada por meio de questionário evidenciou que em relação ao domínio físico, os profissionais perceberam uma piora na qualidade do sono, que as necessidades fisiológicas em geral foram menos atendidas e que o bem-estar geral também foi prejudicado desde o início da pandemia.

No domínio psicológico, houve a diminuição da motivação geral. Isso pode ter sido ocasionado em virtude do cansaço físico, psicológico e de toda pressão enfrentada em relação a assistência e protocolos para o cuidado dos pacientes e de si mesmos. Outro aspecto a ser salientado é uma pequena diminuição da motivação para trabalhar.

Ainda no domínio psicológico, houveram aspectos que melhoraram desde o início da pandemia. Os respondentes demonstraram que tem mais liberdade de expressão e que se sentem orgulhosos, reconhecidos e valorizados no exercício de sua profissão.

No domínio profissional, a qualidade das relações com superiores e subordinados diminuiu, assim como a liberdade para criar coisas novas, a variedade de tarefas realizadas e a igualdade de tratamento entre funcionários. Entretanto, também houveram aspectos que melhoraram. Pois, os respondentes perceberam que seus familiares estão mais orgulhosos de suas profissões, o respeito entre colegas melhorou, há mais participação nas decisões da empresa, maior nível de responsabilidade no trabalho, os treinamentos são melhores, as pessoas estão mais camaradas e o orgulho em relação ao local de trabalho também aumentou. Apesar de diversos pontos positivos, no entanto a qualidade de vida no trabalho caiu consideravelmente.

Este estudo contribui ao trazer um panorama da qualidade de vida dos profissionais de fisioterapia que atuaram em hospitais durante a primeira fase da pandemia de COVID-19. Além disso, pode fornecer insights a respeito de como os profissionais da classe foram impactados pela crise de saúde pública. Entende-se também que, apesar de terem sido prejudicados em sua qualidade de vida no trabalho, também tiveram seus serviços reconhecidos pela população de forma geral. Esse reconhecimento ocorreu, além dos aspectos sociais, por meio da fixação do piso salarial de acordo com o Projeto de Lei n. 1231/2021. 
Dentre as limitações da pesquisa podem ser citadas: baixo número de respondentes e as dificuldades impostas pela inexistência de um cadastro de profissionais que trabalham em hospitais. Assim, ficam como sugestões de pesquisas futuras a análise da qualidade de vida nas diferentes ondas de contaminação da COVID-19 e estudos comparativos com outras regiões adotando o mesmo questionário deste estudo.

\section{Referências}

Almeida, I. M. (2020) Proteção da saúde dos trabalhadores da saúde em tempos de COVID-19 e respostas à pandemia. Rev. bras. saúde ocup. 45, https://doi.org/10.1590/scielopreprints.140.

Andrade, L. S. et al. (2018). Avaliação da qualidade de vida em profissionais de fisioterapia hospitalar intensiva. Cadernos de educação, saúde e fisioterapia, 5(10). https://doi.org/10.18310/2358-8306.v5n10suple

Antunes, E. V., Stefano, S. R. \& Berlatto, H. (2013) A motivação para o trabalho e sua importância estratégica: análise da perspectiva individual em uma empresa do ramo de auto peças e serviços In: IV Encontro de Gestão de Pessoas $e$ Relações de Trabalho, <http://www.anpad.org.br/admin/pdf/EnGPR181.pdf>.

Araújo, A. A., Brito, A. M. \& Novaes, M. (2008). Saúde e autonomia: novos conceitos são necessários? Revista Bioética, 16 , e1, 117 - 24. https://revistabioetica.cfm.org.br/index.php/revista_bioetica/article/view/60

ASSOBRAFIR - Associação Brasileira de Fisioterapia Respiratória. (2020). Fisioterapia na atenção prismática à saúde: recomendações para a atuação dos fisioterapeutas no âmbito da atenção primária à saúde (APS) de pacientes suspeitos ou diagnosticados com COVID-19. de https://assobrafir.com.br/wpcontent/uploads/2020/06/ASSOBRAFIR_COVID-19_APS_2020.06.01.pdf

Belarmino, A. C. et al. (2020). Práticas colaborativas em equipe de saúde diante da pandemia de COVID-19. Rev. Bras. Enferm., 73(2), e20200470. https://doi.org/10.1590/0034-7167-2020-0470.

Brasil. (2012). Ministério da Saúde: Resolução $n^{o} 466$, de 12 de dezembro de $2012 . \quad$ Conselho Nacional de Saúde 2012. http://bvsms.saude.gov.br/bvs/saudelegis/cns/2013/res0466_12_12_2012.html

Craigie, M. et. al. (2016). The Influence of Trait-Negative Affect and Compassion Satisfaction on Compassion Fatigue in Australian Nurses. American Psychological Association, 8(1), 88-97. https://doi.org/10.1037/tra0000050 .

Cruz, D. S. M., Collet, N. \& Nóbrega, V. M. (2018). Qualidade de vida relacionada à saúde de adolescentes com dm1- revisão integrativa. Ciênc. saúde colet. 23(3), 973-989. https://doi.org/10.1590/1413-81232018233.08002016.

Esperidião, E., Saidel, M. G. B., Rodrigues, J. (2020). Saúde mental: foco nos profissionais de saúde. Rev. Bras. Enferm. 73(1), e73supl01. https://doi.org/10.1590/0034-7167.202073supl01.

Faro, A. et al. (2020). COVID-19 e saúde mental: a emergência do cuidado. Estudos de Psicologia (Campinas) 37, e200074. https://doi.org/10.1590/1982$0275202037 \mathrm{e} 200074$

Ferro, F. F. (2012). Instrumentos para medir a qualidade de vida no trabalho e a ESF: uma revisão de literatura. Monografia (Especialização em Atenção Básica a Saúde da Família). Belo Horizonte: Universidade Federal de Minas Gerais http://www.nescon.medicina.ufmg.br/biblioteca/imagem/3756.pdf

Gordia, A. P. et al. (2015). Domínio social da qualidade de vida de adolescentes e sua associação com variáveis comportamentais, biológicas e sociodemográficas. Rev. educ. fis. UEM, 26(3), 451-463. http://dx.doi.org/10.4025/reveducfis.v26i3.23066.

Guimarães, F. (2020). Atuação do fisioterapeuta em unidades de terapia intensiva no contexto da pandemia de COVID-19. Fisioter. Mov. 33, http://dx.doi.org/10.1590/1980-5918.033.ED01.

Helioterio, M. C. et al. (2020). COVID-19: por que a proteção da saúde dos trabalhadores e trabalhadoras da saúde é prioritária no combate à pandemia? Trabalho, Educação e Saúde, 18(3), e00289121. https://doi.org/10.1590/1981-7746-sol00289.

IBSP - Instituto Brasileiro de Segurança do Paciente. (2020). COVID-19 - Em momentos de pandemia é preciso proteger os profissionais da saúde. https://www.segurancadopaciente.com.br/protocolo-diretrizes/covid-19-em-momentos-de-pandemia-e-preciso-proteger-os-profissionais-da-saude

Karsten, M., Matte, D. L., Andrade, F. M. D. (2020). The COVID-19 pandemic brought challenges and new possibilities for Physiotherapy in Brazil: are we ready? J. Physiother. Res., Salvador, 10(2), 142-145. https://doi.org/10.17267/2238-2704rpf.v10i2.2971

Lotta, G. et al. (2020). A pandemia de Covid-19 e os(as) agentes prisionais/policiais penais no Brasil. Nota Técnica. Fundação Getúlio Vargas, Núcleo de Estudos da Burocracia (NEB). https://www.researchgate.net/profile/Gabriela_Lotta/publication/343538059_Impactos_da_Covid-19_nos_agentes_prisionais__2a_fase/links/5f2fdc21a6fdcccc43b88399/Impactos-da-Covid-19-nos-agentes-prisionais-2a-fase.pdf.

Maissiat, G. S. et al. (2015). Contexto de trabalho, prazer e sofrimento na atenção básica em saúde. Rev Gaúcha Enferm. 36(2), 42-49. http://dx.doi.org/10.1590/1983-1447.2015.02.51128.

Mendonça, A. E. O. et al . (2015). Análise dos aspectos físicos da qualidade de vida de receptores de rim. Rev. esc. enferm. USP, 49(1), 76-81. https://doi.org/10.1590/S0080-623420150000100010.

Oliveira, W. A. et al. (2020). Impactos psicológicos e ocupacionais das sucessivas ondas recentes de pandemias em profissionais da saúde: revisão integrativa e lições aprendidas. Estud. psicol. (Campinas), 37, e200066. https://doi.org/10.1590/1982-0275202037e200066. 
OMS. (1995). The World Health Organization Quality of Life assessment (WHOQOL): position paper from the World Health Organization. Soc Sci Med, 41(10), 1403-1409. https://doi.org/10.1016/0277-9536(95)00112-k

Ornell, F. et al. (2020). O impacto da pandemia de COVID-19 na saúde mental dos profissionais de saúde. Saúde Pública, 36(4), e00036520, http://dx.doi.org/10.1590/0102-311X00036520.

Paz, M. G. T. Personal organizational well-being and quality of organizational life: the mediating role of organizational culture. RAM. Revista de Administração Mackenzie [online]. 21(1), eRAMD200122. https://doi.org/10.1590/1678-6971/eRAMD200122.

Pereira, E. F., Teixeira, C. S. \& Santos, A. (2012). Qualidade de vida: abordagens, conceitos e avaliação. Rev. bras. educ. fís. esporte, 26(2), 241-250. https://doi.org/10.1590/S1807-55092012000200007.

Peruzzo, H. E., Bega, A. G. \& Lopes, A. P. A. T. (2018). Os desafios de se trabalhar em equipe na estratégia saúde da família. Esc. Anna Nery, 22(4), http://dx.doi.org/10.1590/2177-9465-ean-2017-0372.

Ravagnani, J. S., Crivelato, P. M. S. (2010). Qualidade de Sono e Percepção da Qualidade de Vida dos Profissionais de Enfermagem de uma Unidade de Terapia Intensiva. Trabalho de Conclusão de Curso (Graduação em Enfermagem). Centro Universitário Católico Salesiano, http://www.unisalesiano.edu.br/biblioteca/monografias/51939.pdf

Reis Jr., D. R. (2008). Qualidade de Vida no Trabalho: construção e validação do questionário QWLQ-78. Dissertação (Mestrado em Engenharia de Produção), Universidade Tecnológica Federal do Paraná. Ponta Grossa, Paraná.

Reis Jr., D. R., Pilatti, L. A. \& Pedroso, B. (2011). Qualidade de vida no trabalho: construção e validação do questionário QWLQ-78. Revista Brasileira de Qualidade de Vida, 3(2), 1-12. http://www.doi.org/10.3895/S2175-08582011000200001

Ribeiro, A. P., Oliveira, G. L., Silva, L. S. et al. (2020). Saúde e segurança de profissionais de saúde no atendimento a pacientes no contexto da pandemia de Covid-19: revisão de literatura. Rev. bras. saúde ocup. 45, e. 25. https://doi.org/10.1590/2317-6369000013920.

Sampaio, J. R. (2012). Qualidade de vida no trabalho: perspectivas e desafios atuais. Rev. Psicol., Organ. Trab., 12(1), 121-136. http://pepsic.bvsalud.org/scielo.php?script=sci_arttext\&pid=S1984-66572012000100011\&lng=pt\&nrm=iso

Santana, V. S., Feitosa, A. G., Arruda Guedes, L. B., \& Brito Sales, N. B. (2014). Qualidade de vida dos profissionais de saúde em ambiente hospitalar. Revista Pesquisa Em Fisioterapia, 4(1), 35-46. https://doi.org/10.17267/2238-2704rpf.v4i1.312

Schramm, F. R. et al. (2020). O aparente dilema implicado pela pandemia da COVID-19: salvar vidas ou a economia? Observatório Covid-19 Fiocruz. https://www.arca.fiocruz.br/handle/icict/41374.

Silva, C. M. S. et al. (2020). Evidências científicas sobre Fisioterapia e funcionalidade em pacientes com COVID-19 Adulto e Pediátrico. Rev. Bras. Crescimento Desenvolv. Hum. 30(1), http://doi.org/10.7322/jhgd.v30.10086

Silva, R. M. V.; Sousa, A. V. C. (2020). Fase crônica da COVID-19: desafios do fisioterapeuta diante das disfunções musculoesqueléticas. Fisioter. mov., 33, e0033002. http://dx.doi.org/10.1590/1980-5918.033.ed02.

Socorro F. H. O. S. et al. (2020). As funções da equipe pluridisciplinar no cuidado da covid-19. Braz. J. Hea. Rev., Curitiba, 3(5), 17577-12591. https://dx.doi.org/10.34119/bjhrv3n5-098.

Soeiro, R. E. et al. (2020). Atenção Primária à Saúde e a pandemia de COVID-19: reflexão para a prática. InterAmerican Journal of Medicine and Health, 3. https://doi.org/10.31005/iajmh.v3i0.83

Spiller, A. P. M., Dyniewicz, A. M. \& Slomp, M. G. (2008). Qualidade de vida de profissionais da saúde em hospital universitário. Cogitare Enfermagem, v. 13, n. 1, pp. 88-95. Recuperado em 26 de novembro de 2021, de https://www.redalyc.org/pdf/4836/483648978012.pdf

Sumiya, A. (2020). Educação, Saúde e Fisioterapia em tempos de COVID-19. Caderno Educação, Saúde e Fisioterapia. 7(13), https://doi.org/10.18310/23588306.v7n13.ed1

Teixeira, C. F. S. et al. (2020). A saúde dos profissionais de saúde no enfrentamento da pandemia de Covid-19. Ciênc. saúde coletiva, 25(9), 3465-3474 https://doi.org/10.1590/1413-81232020259.19562020.

Valle, L. E. L. R, Valle, E. L. R. \& Reimão, R. (2009). Sono e aprendizagem. Revista Psicopedagogia, 26(80), 286-290. http://pepsic.bvsalud.org/scielo.php?script=sci_arttext\&pid=S0103-84862009000200013\&lng=pt\&tlng=pt. 